\title{
A criterion for a set to be of 1-dimensional measure zero
}

\author{
By Tadashi KurodA
}

(Received August 22, 1959)

1. Let $F$ be a domain in the complex $z$-plane $\Omega$ and let $E$ be the boundary of $F$. We assume that the point $z=\infty$ is an interior point of $F$. Consider a finite number of doubly connected domains $R^{(k)}(k=1, \cdots, \nu)$ satisfying the followings:

i) the closure $\overline{R^{(k)}}$ of $R^{(k)}$ is contained in $F$,

ii) the boundary of $R^{(k)}$ consists of two analytic closed curves,

iii) the complementary set of each $\overline{R^{(k)}}$ consists of two domains the one $F^{(k)}$ of which contains the point $z=\infty$ and the other $G^{(k)}$ of which has at least a point common with the set $E$, and

iv) any point of the set $E$ is contained in some $G^{(k)}$

v) if $i \neq j$, then $R^{(i)}$ lies in $F^{(j)}$.

We shall say that the system $R=\left\{R^{(k)}\right\}_{k=1}^{\nu}$ of such doubly connected domains separates, in $F$, the point $z=\infty$ from $E$. If we put $F_{0}=\bigcap_{k=1}^{\nu} F^{(k)}$, we can see that $F_{0}$ is a domain compact relative to $F$ and that the complementary set of the set $\bigcup_{k=1}^{\nu} \overline{R^{(k)}} \cup F_{0}$ is the union $\bigcup_{k=1}^{\nu} G^{(k)}$.

We can always take an enumerable number of systems $R_{n}=\left\{R_{n}^{(k)}\right\}_{k=1}^{\nu(n)}(n=1,2, \cdots)$ of doubly connected domains such that each system $R_{n}$ separates, in $F$, the point $z=\infty$ from the boundary of $F$ and that each domain $R_{n+1}^{(k)}$ of the system $R_{n+1}$ lies in a domain $G_{n}^{(l)}$ being a connected component of the complementary set of some $\overline{R_{n}^{(2)}}$ and not containing the point $z=\infty$. In the following, systems $R_{n}(n=1,2, \cdots)$ separating, in $F, z=\infty$ from $E$ means such a sequence of systems as above.

Now, denote by $\log \mu_{n}^{k}$ the modulus of the domain $R_{n}^{(k)}$ and put

$$
\log \mu_{n}=\operatorname{Min}_{1 \leqq k \leqq \nu(n)} \log \mu_{n}^{k} .
$$

Considering a boundary component $\gamma$ of $F$ in the sense of Kerékjártó-Stoïlow, we see that there exists a sequence of domains $R_{n}^{\left(k_{n}\right)}(n=1,2, \cdots)$ such that the domain $G_{n}^{\left(k_{n}\right)}$ contains $\gamma$ and $R_{m}^{\left(k_{m}\right)}$ for any $m(>n)$. If the series

$$
\sum_{n=1}^{\infty} \log \mu_{n}
$$

diverges, then the series

$$
\sum_{n=1}^{\infty} \log \mu_{n}^{k} n
$$

is also divergent and, hence, by Savage's theorem [6], the boundary component $\gamma$ is weak in the sense of Sario [5]. Therefore, we have easily the following proposition from Sario's theorem [5].

Proposition 1. If the series (1) diverges, then the boundary set $E$ of $F$ belongs to $N_{S_{B}}$ in the sense of Ahlfors-Beurling [1]. 
Of course, the above proposition is a direct corollary of the following due to Sario [4].

Proposition 2. If the series (1) diverges, then the set $E$ belongs to $N_{\mathfrak{I}}$.

Further, by Pfluger-Mori's result [2], [3], we can easily verify the following

Proposition 3. If

$$
\limsup _{m \rightarrow \infty}\left[\sum_{n=1}^{m} \log \mu_{n}-\frac{1}{2} \log \nu(m)\right]=+\infty
$$

then the set $E$ belongs to $N_{\mathfrak{B}}$.

2. Here we shall give a criterion for a linear set $E$ to belong to $N_{\mathfrak{B}}$ which depends on moduli $\log \mu_{n}$ but is independent of the number $\nu(n)$. We use the same notations as in $\S \mathbf{1}$.

THEOREM. Let $E$ be a closed set lying on a straight line $L$. If there exist systems $R_{n}(n=1,2, \cdots)$ separating $z=\infty$ from $E$ such that

a) each $R_{n}^{(k)}(1 \leqq k \leqq \nu(n), n=1,2, \cdots)$ is bounded by two circles symmetric about $L$ and that

b) the series (1) diverges, then the set $E$ is of 1-dimensional measure zero and hence belongs to $N_{\mathfrak{B}}$.

To establish this, we use the following elementary lemma.

LEMMA. Let $\Delta$ be a doubly connected domain bounded by two circles $|z|=\rho$ and $\left|z-z_{0}\right|=r$, where $z_{0}$ is real and $\operatorname{Max}\left(\left|z_{0}+r\right|,\left|z_{0}-r\right|\right)<\rho$. Then the modulus of $\Delta$ is dominated by $\log (\rho / r)$ from the above.

Proof. It is easy to see that the circle $\left|z-z_{0}\right|=r$ lies in $|z|<\rho$. By a suitable linear transformation

$$
\zeta=\zeta(z)=\rho \frac{z-\alpha \rho}{\rho-\alpha z} \quad(-1<\alpha<1)
$$

we can map $\Delta$ in the concentric ring domain $\delta<|\zeta|<\rho$ one to one conformally such that the images of points $z_{1}=-\rho, z_{2}=z_{0}-r, z_{3}=z_{0}+r$ and $z_{4}=\rho$ are the points $\zeta_{1}=-\rho, \zeta_{2}=-\delta, \zeta_{3}=\delta$ and $\zeta_{4}=\rho$, respectively, where $\delta>0$. It is evident that the cross ratio $\left(z_{1}, z_{2}, z_{3}, z_{4}\right)$ equals the cross ratio $\left(\zeta_{1}, \zeta_{2}, \zeta_{3}, \zeta_{4}\right)$. From this, we have

$$
\frac{(\rho+\delta)^{2}}{\delta}=\frac{\left(r+\rho+z_{0}\right)\left(r+\rho-z_{0}\right)}{r} .
$$

Hence we get the inequality

$$
\frac{\rho^{2}}{\delta}+\delta \leqq \frac{\rho^{2}}{r}+r
$$

Since the function $\left(\rho^{2} / x\right)+x$ is monotonously decreasing in $0<x<\rho$, we obtain the inequality $\delta \geqq r$. The modulus of $\Delta$ equals $\log (\rho / \delta)$, so we have our lemma.

Proof of Theorem. We denote by $C_{n}^{(k)}$ and $\Gamma_{n}^{(k)}$ the interior circle and the exterior circle bounding $R_{n}^{(k)}$, respectively. Let $r_{n}^{(k)}$ and $\rho_{n}^{(k)}$ be the radius of $C_{n}^{(k)}$ and $\Gamma_{n}^{(k)}$, respectively, and put 


$$
r_{n}=\sum_{k=1}^{\nu(n)} r_{n}^{(k)}, \quad \rho_{n}=\sum_{k=1}^{\nu(n)} \rho_{n}^{(k)}
$$

It is evident that $r_{n} \geqq \rho_{n+1}$. Since the closed discs $\left(\Gamma_{n}^{(k)}\right)$ bounded by $\Gamma_{n}^{(k)}(k=1, \cdots$, $\nu(n))$ cover the set $E$, it is sufficient to show that $\lim _{n \rightarrow \infty} \rho_{n}=0$. By our lemma, we have

$$
\log \mu_{n}=\operatorname{Min}_{1 \leqq k \leqq \nu(n)} \log \mu_{n}^{k} \leqq \log \rho_{n}^{(k)}-\log r_{n}^{(k)}
$$

or

$$
\mu_{n} r_{n}^{(k)} \leqq \rho_{n}^{(k)} \quad(k=1, \cdots, \nu(n)) .
$$

Summing up these, we get

$$
\mu_{n} r_{n} \leqq \rho_{n}
$$

which gives

$$
\mu_{n} \leqq \frac{\rho_{n}}{r_{n}} \leqq \frac{\rho_{n}}{\rho_{n+1}}
$$

Therefore, it holds

$$
\prod_{n=1}^{N} \mu_{n} \leqq \frac{\rho_{1}}{\rho_{N+1}} .
$$

By the assumption b), we have $\lim _{n \rightarrow \infty} \rho_{n}=0$.

3. In the case of a special domain $F$, we can prove the converse of our Theorem.

Consider a segment $S_{1}$ with length $l_{1}$ on the real axis of $\Omega$. From $S_{1}$, we delete the open segment $T_{1}$ with the length $l_{1} / p_{1}\left(p_{1}>1\right)$ such that the set $S_{2}=S_{1}-T_{1}$ consists of two closed segments $S_{2}^{(j)}(j=1,2)$ with equal length $l_{2}$. Evidently the total length of $S_{2}$ equals $2 l_{2}=l_{1}\left(1-1 / p_{1}\right)$. In general, from the set $S_{m}$ we delete the open segments $T_{m}^{(j)}\left(j=1, \cdots, 2^{m-1}\right)$ such that each $T_{m}^{(j)}$ has length $l_{m} / p_{m}\left(p_{m}>1\right)$ and that $S_{m}-\cup_{j=1}^{2^{m-1}} T_{m}^{(j)}$ consists of closed segments $S_{m+1}^{(j)}\left(j=1, \cdots, 2^{m}\right)$ with equal length $l_{m+1}$. It is obvious that the total length of the set $S_{m}$ is $2^{m-1} l_{m}=2^{m-2} l_{m-1}(1$ $\left.-1 / p_{m-1}\right)=l_{1} \prod_{k=1}^{m-1}\left(1-1 / p_{k}\right), \quad S_{m} \supset S_{m+1}$ and $\cap_{m=1}^{\infty} S_{m}$ is a non-empty perfect closed set. We denote this set by $E\left(p_{1}, p_{2}, \cdots\right)$ and call this a Cantor set. Obviously, the complementary set $F$ of $E\left(p_{1}, p_{2}, \cdots\right)$ with respect to $\Omega$ is a domain. We put two assumptions:

$\alpha)$

$$
2 p_{k+1}>p_{k}-1 \quad(k=1,2, \cdots)
$$

and

$$
\prod_{k=1}^{\infty}\left(1-\frac{1}{p_{k}}\right)=0 .
$$

The assumption $\alpha$ ) is not so strong. For instance, if $p_{k} \leqq p_{k+1}(k=1,2, \cdots)$, then $\alpha)$ is satisfied. The assumption $\beta$ ) is equivalent to the assumption that the set $E\left(p_{1}, p_{2}, \cdots\right)$ is of 1 -dimensional measure zero.

We describe two concentric circles $\Gamma_{m+1}^{(j)}$ and $C_{m+1}^{(j)}$ with centre $z_{m+1}^{(j)}$ being the middle point of $S_{m+1}^{(j)}(m \geqq 1)$ and with the radius

$$
\rho_{m+1}=\frac{l_{m}}{4}-\left(1+\frac{1}{p_{m}}\right)
$$

and the radius 


$$
r_{m+1}=\frac{l_{m}}{4}\left(1+\frac{1}{p_{m+1}}\right)\left(1-\frac{1}{p_{m}}\right),
$$

respectively. Then, since

we see by the assumption $\alpha$ )

$$
l_{m+1}=\frac{1}{2} l_{m}\left(1-\frac{1}{p_{m}}\right)
$$

$$
l_{m+1}<2 r_{m+1}<2 \rho_{m+1}=r_{m} .
$$

In other words, the circle $C_{m+1}^{(j)}$ lies inside $\Gamma_{m+1}^{(j)}$ and the segment $S_{m+1}^{(j)}$ lies inside $C_{m+1}^{(j)}$. If we denote by $R_{m}^{(j)}$ the doubly connected domain bounded by $C_{m+1}^{(j)}$ and $\Gamma_{m+1}^{(j)}$, the systems $R_{m}=\left\{R_{m}^{(j)}\right\}_{j=1}^{2 m}(m=1,2, \cdots)$ separates, in $F, z=\infty$ from $E\left(p_{1}, p_{2}, \cdots\right)$ and satisfies the condition a) of Theorem.

Next, the modulus $\log \mu_{m}^{j}$ of $R_{m}^{(j)}\left(j=1, \cdots, 2^{m}\right)$ is equal to

Thus we get

$$
\log \frac{\rho_{m+1}}{r_{m+1}}=\log \left(1+\frac{1}{p_{m}}\right)-\log \left(1+\frac{1}{p_{m+1}}\right)\left(1-\frac{1}{p_{m}}\right) \text {. }
$$

$$
\begin{aligned}
\sum_{m=1}^{N} \log \mu_{m}= & \log \prod_{m=1}^{N} \frac{\left(1+\frac{1}{p_{m}}\right)}{\left(1+\frac{1}{p_{m+1}}\right)\left(1-\frac{1}{p_{m}}\right)} \\
& =\log \frac{1+\frac{1}{p_{1}}}{1+\frac{1}{p_{N+1}}} \frac{1}{\prod_{m=1}^{N}\left(1-\frac{1}{p_{m}}\right)} \geqq \log \frac{1}{2 \prod_{m=1}^{N}\left(1-\frac{1}{p_{m}}\right)} .
\end{aligned}
$$

By the assumption $\beta$ ), the right hand side of this inequality diverges as $N$ tends to infinity and hence we obtain the condition b) of Theorem. Thus we have

Profosition 4. For a Cantor set $E\left(p_{1}, p_{2}, \cdots\right)$ satisfying $\left.\alpha\right)$ to be of 1-dimentional measure zero, it is necessary and sufficient that there exist systems $R_{n}=\left\{R_{n}^{(k)}\right\}_{k=1}^{\nu(n)}$ $(n=1,2, \cdots)$ separating, in $F=\Omega-E\left(p_{1}, f_{2}, \cdots\right)$, the point $z=\infty$ from $E\left(p_{1}, p_{2}, \cdots\right)$ and satisfying the conditions a) and b) in our Theorem.

REMARK. An analogue of Proposition 4 is obtained for some special 2-dimensional Cantor set $E^{2}\left(p_{1}, p_{2}, \cdots\right)$ (Cf. Sario [4]). Proposition 4 and this analogue are closely related to results of Sario [4].

\section{Mathematical Institute, \\ Nagoya University.}

\section{References}

[1] Ahlfors, L., and A. Beurling, Conformal invariants and function-theoretic null sets. Acta Math. 83 (1950), 101-129.

[2] Mori, A., A note on unramified abelian covering surfaces of a closed Riemann surface. Journ. Math. Soc. Jap. 6 (1954), 162-176.

[3] Pfluger A., Sur l'existence de functions non constantes, analytiques, uniformes et bornées sur une surface de Riemann ouverte. C. R. Paris 230 (1950), 166-168.

[4] Sario, L., Über Riemannsche Flächen mit hebbarem Rand. Ann. Acad. Sci. Fenn., A. I., 50 (1948).

[5] Sario, L., Capacity of the boundary and a boundary component. Ann. of Math. 59 (1954), 135-144.

[6] Savage, N., Weak boundary components of an open Riemann surface. Duke Math. Journ. 24 (1957), 79-95. 